\title{
Mitochondrial genome function and maternal inheritance
}

\author{
John F. Allen" $\dagger^{1}$ and Wilson B.M. de Paula* \\ "School of Biological and Chemical Sciences, Queen Mary University of London, Mile End Road, London E1 4NS, U.K., and †Research Department of Genetics, \\ Evolution and Environment, University College London, Gower Street, London WC1E 6BT, U.K.
}

\begin{abstract}
The persistence of mtDNA to encode a small subset of mitochondrial proteins reflects the selective advantage of co-location of key respiratory chain subunit genes with their gene products. The disadvantage of this colocation is exposure of mtDNA to mutagenic ROS (reactive oxygen species), which are by-products of aerobic respiration. The resulting 'vicious circle' of mitochondrial mutation has been proposed to underlie aging and its associated degenerative diseases. Recent evidence is consistent with the hypothesis that oocyte mitochondria escape the aging process by acting as quiescent genetic templates, transcriptionally and bioenergetically repressed. Transmission of unexpressed mtDNA in the female germline is considered as a reason for the existence of separate sexes, i.e. male and female. Maternal inheritance then circumvents incremental accumulation of age-related disease in each new generation.
\end{abstract}

\section{Mitochondria and their genomes}

Mitochondria are cytoplasmic organelles of eukaryotes. Their most conspicuous role in cellular metabolism is respiration, which includes the stepwise oxidation of imported pyruvate, release of carbon dioxide and the coupling of electron transport to synthesis of ATP in oxidative phosphorylation. In many eukaryotes, the terminal respiratory electron acceptor is oxygen. Aerobic respiration takes place in mitochondria containing a small specialized genome and a complete apparatus of gene expression, distinct from that of the cell nucleus and cytosol.

The genomes of mitochondria contain a minute subset of genes [1] retained from their alphaproteobacterial ancestors, with other genes of prokaryotic endosymbiont origin either having been lost or else retained as genes now found only on chromosomes of the cell nucleus [2]. Genes in mitochondria always encode mitochondrial proteins or RNAs (reviewed in [3]); their gene products are not exported. A single mitochondrion contains more than a thousand proteins [4-6], perhaps as many as its bacterial ancestor, and the great majority are imported from the cytosol as precursors, subsequently processed $[7,8]$. In the mitochondrial inner membrane, the apparatus of oxidative phosphorylation includes respiratory chain complexes with nuclearly encoded imported subunits as well mitochondrially encoded subunits synthesized on ribosomes in the mitochondrial matrix $[9,10]$.

If the overwhelming majority of mitochondrial proteins are now encoded in the nucleus for synthesis in the cytoplasm, then why not all? Why has mtDNA been retained? What is

Key words: CoRR hypothesis, female germline, mitochondrial DNA (mtDNA), mitochondrial transcription, promitochondrion.

Abbreviations used: CoRR, co-location for redox regulation; CSK, chloroplast sensor kinase; ROS, reactive oxygen species.

${ }^{1}$ To whom correspondence should be addressed (email j.f.allen@qmul.ac.uk). it about the genes encoded there, or their gene products, that demands a separate, dedicated, cytoplasmic genetic system in a primarily energy-transducing organelle?

\section{CoRR (co-location for redox regulation)}

One hypothesis for the evolution and retention of the cytoplasmic genomes in bioenergetic organelles, both mitochondria and chloroplasts, was proposed in 1993 [11], and later termed CoRR for co-location (of gene and gene product) for redox regulation (of gene expression) [12,13].

The central proposition of the CoRR hypothesis is that regulation of gene expression by the redox state of electron carriers existed in the bacterial ancestors of mitochondria and chloroplasts, that this regulation continued, uninterrupted, throughout the transition from free-living prokaryote to eukaryotic organelle, and that it remains in operation today. CoRR proposes that genes that are subject to direct redox regulatory control remain in the same subcellular compartment as their gene products. The relative abundance, or stoichiometry, of these gene products is thereby rapidly and unconditionally adjusted in response to environmental changes in availability of electron sources and sinks, and to changes in energetic and metabolic supply and demand. CoRR further proposes that mitochondria and chloroplasts retain formerly bacterial redox signalling pathways that facilitate the required regulatory coupling between electron transport and gene expression. Genes for components of these signalling pathways are predicted to be encoded in the nucleus, since they encode part of the major class of organellar proteins and have no need, themselves, to be subject to redox regulatory control.

Subsequent research, specifically on chloroplast gene expression, is broadly consistent with the CoRR hypothesis. There is evidence in favour of chloroplast CoRR in 
four independent ways: (i) chloroplast transcription is differentially regulated by the redox state of a specific component of the photosynthetic electron transport chain [14]; (ii) the effect of this regulation is to adjust the stoichiometry of chloroplast Photosystem I and Photosystem II, restoring the redox poise of the plastoquinone pool after it has been perturbed by transient changes in light quality, or by site-specific inhibition of electron transport $[14,15]$; (iii) a cyanobacterial redox sensor kinase, part of a twocomponent regulatory system, is retained in chloroplasts of plants and eukaryotic algae [16]; and (iv) this CSK (chloroplast sensor kinase) is required for redox regulation of photosynthetic reaction centre gene transcription, and is encoded in the nucleus [16,17]. Further experiments indicate that the redox transcriptional control pathway involving CSK $[18,19]$ and $\sigma$ factor-1 [20] has been partially rewired: although it still terminates with a plastid-encoded RNA polymerase, chloroplast redox signalling also governs the action of a protein kinase of eukaryotic origin $[17,21]$.

If the CoRR hypothesis has led to better understanding of the evolution, function and regulation of the chloroplast genome, what is the result of its application to mitochondria?

\section{Does CoRR apply to mitochondria too?}

Evidence compatible with CoRR includes effects of redox reagents and electron transport inhibitors on qualitative patterns of protein synthesis, probed by $\left[{ }^{35} \mathrm{~S}\right]$ methionine labelling, both in isolated mitochondria and in isolated chloroplasts [22,23]. Subsequent experiments with plant mitochondria implicate respiratory complex II, which is usually alone in being a wholly nuclearly encoded respiratory complex, as the site of redox control [24]. This conclusion is consistent with the key cofactor being ubiquinone, the mitochondrial analogue of chloroplast plastoquinone. Redox control of mitochondrial RNA synthesis has also been reported [25].

Independent work on mitochondria, not addressing the CoRR hypothesis directly, nevertheless fits with the prediction that a mitochondrial genome is required in order to exert regulatory control over respiratory electron transport. Mitochondria that have lost oxidative phosphorylation have also lost their genomes. Such relict mitochondria $[26,27]$ include mitosomes of anaerobic protists such as Entamoeba histolytica [28] and Giardia lamblia [29], which are proposed to retain a secondary mitochondrial function, iron-sulfur cluster biogenesis [29,30], as their contribution to biosynthesis and metabolism. Hydrogenosomes [31,32] carry out energy transduction, but have lost membrane-intrinsic complexes of oxidative phosphorylation. Hydrogenosomes have, accordingly, no genomes of their own $[27,33]$. Diverse mitochondrial forms and functions are retained in various cell types and eukaryotic lineages [34]. We suggest that retention of a mitochondrial genome may correlate with retention of oxidative phosphorylation, and that transmission of mitochondrial genomes acquires an additional constraint in fully aerobic animals and plants [26,35]. Intracellular compartmentalized energy transduction may depend on CoRR, and have given rise to the lower energetic cost of gene expression and replication underlying the complexity of eukaryotic cells, with their ability to differentiate and co-operate, allowing multicellularity [36]

A modified two-component redox regulatory system lies at the centre of chloroplast redox control of transcription $[21,37]$. Mitochondrial two-component systems have been described in fungi [38], but not in animals [39]. A singlecomponent redox regulator or activator can transmit redox information to regulate transcription in bacteria (reviewed in [40]) and could, on its own, satisfy the requirement of CoRR. The Hydrogen Hypothesis for the origin of mitochondria and eukaryotes [41] envisages a metabolically and genetically flexible ancestral alphaproteobacterium, giving rise to each of the different types of mitochondria [34] by selective loss of function. Aerobic mitochondria of animals may therefore retain redox signalling systems, as yet incompletely characterized. Aconitase [42] and topoisomerase I [43], for example, are implicated in mitochondrial redox signalling.

\section{Adjustment of respiratory chain complex stoichiometry by means of ubiquinone redox control of mitochondrial gene transcription}

The chloroplast gene regulatory system relies on a recognizably bacterial-type transcriptional machinery, whereas many mitochondria may have lost parts of such a system during their evolution, replacing them with phage-type components [44]. We suggest that mitochondria have rewired bacterial redox regulatory components in order to maintain ubiquinone redox balance. This mechanism may include phosphorylation of a phage-type RNA polymerase integrating mitochondrial transcription factors with a mitochondrial redox sensor kinase, rather than phosphorylation of bacterialtype $\sigma$ factors, as seen for chloroplasts [21].

According to the specific scheme envisaged in Figure 1, an elevated ratio of ubiquinol to ubiquinone inhibits the kinase activity of a mitochondrial ubiquinone redox sensor, and, as a consequence, mitochondrial transcription factors become free to bind to mtDNA and to facilitate the transcription of genes for core subunits of its primary and secondary electron acceptors, complexes III and IV, as shown in Figure 1(a). Increased electron flow out, and decreased electron flow in, then restore optimal redox state of the quinone pool, where ubiquinone (oxidized) and ubiquinol (reduced) are present at a ratio of 1:1. Conversely, a decreased ubiquinol/ubiquinone ratio activates the kinase domain of the proposed mitochondrial redox sensor. This in turn phosphorylates mitochondrial transcription factors, leading to transcriptional suppression of the complex III and IV core subunit genes. The proposed effect of this redox control, indicated in Figure 1(b), will be decreased electron flow out, and increased electron flow in, again restoring ubiquinone redox poise. 
Figure 1| Proposed regulatory control of mitochondrial transcription by the redox state of the ubiquinol/ubiquione pool

(a) An elevated NADH output from the tricarboxylic acid cycle (1) increases the ubiquinol/ubiquinone ratio (2) and inhibits the kinase activity of the mitochondrial redox sensor (3), which allows mitochondrial transcription factors to bind to mtDNA (4) to promote transcription (5) and subsequent translation (6) of cob and cox1 genes, subsequently increasing the electron flow out toward molecular oxygen as the final acceptor (7). (b) Conversely, if the NADH supply is diminished (1), the ubiquinone pool becomes more oxidized (2). This activates the kinase domain of the mitochondrial redox sensor (3), which in turn phosphorylates the mitochondrial transcription factor, precluding its binding to mtDNA preventing up-regulation of cob and cox1 transcription. In the inner mitochondrial membrane, respiratory complexes I, III and IV are depicted as surface models rendered using PyMOL (http://www.pymol.org) from PDB codes 3M9S, 1QCR and 1V54 respectively.

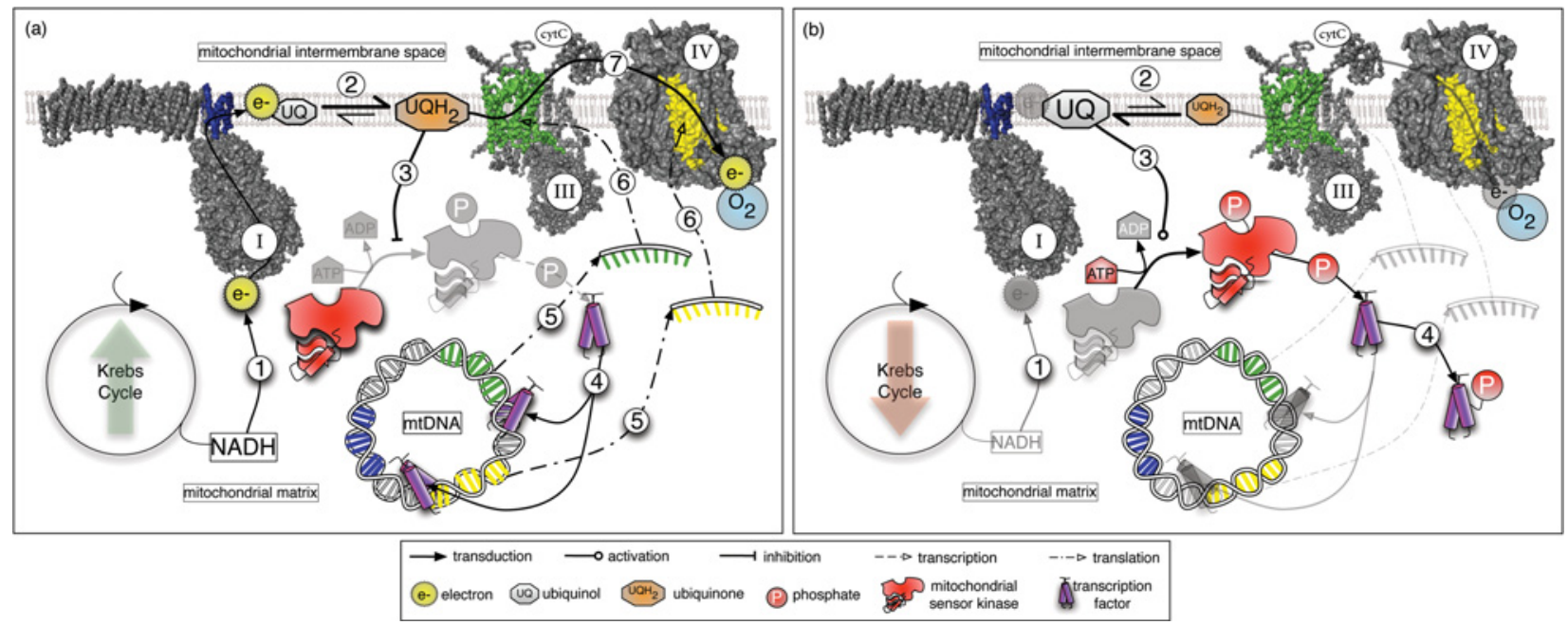

\section{Requirement for redox poise}

A self-regulatory mechanism of the kind shown in Figure 1 may operate even in anaerobic mitochondria, where it may serve to increase efficiency by matching respiratory complex stoichiometry to the kinetics of electron flow. In plants and micro-organisms with branched respiratory chains, a prolonged redox imbalance may also send signals to initiate de novo synthesis of additional or alternative electron donors or acceptors. However, maintaining ubiquinone redox poise may be especially important in the presence of molecular oxygen. The reason for this is that ubisemiquinone, a transient intermediate in complexes I and III, itself carries an unpaired electron, and reacts readily with molecular oxygen to yield the superoxide anion radical $[45,46]$. Superoxide is indiscriminately reactive and mutagenic, and is also a precursor of related and cytotoxic ROS (reactive oxygen species). Therefore a precisely balanced respiratory chain will tend to minimize destructive effects of oxygen chemistry, not only on the chain itself, but also on the genes for its core components. Thus, if there is a benefit in having a mitochondrial genome, there is also, potentially, a serious and long-lasting cost [47] in the form of accumulated mis-coding of mitochondrial apoproteins that are correspondingly more likely to catalyse mutagenic single-electron transfers to oxygen. This 'vicious circle', of DNA damage giving protein damage giving more DNA damage [48], highlights what otherwise amounts to a 'design flaw' in aerobic, eukaryotic cells. Without the benefit of a redox autoregulatory system of the kind depicted in Figure 1, mitochondria are about the worst imaginable location in the cell to store a genetic system. In the absence of CoRR, all formerly endosymbiont genes would be expected to have relocated the metabolically and electrochemically inert compartment represented by the cell nucleus.

\section{The mitochondrial theory of aging}

The mitochondrial theory of aging [49] states that superoxide anions $\left(\mathrm{O}_{2}{ }^{-}\right)$, produced by single-electron transfers from the mitochondrial respiratory chain, react with the DNA retained in the mitochondrial matrix, causing deleterious rearrangements that accumulate over time [50,51]. There is debate over whether ROS are the direct cause of the decline of mitochondrial function seen in aging $[52,53]$, although recent studies are certainly consistent with the theory. Superoxide production increases with age of brain tissue in a senescence-accelerated mouse line, in rats, and in pigeons [54]. Oxidative damage and apoptosis occur during aging in the brain, oculus and kidney of mice and are accompanied by increased $\mathrm{O}_{2}{ }^{-}$production. Comparatively lower $\mathrm{O}_{2}{ }^{-}$concentrations are found in cardiac and muscle tissues [55]. Pressure-induced arterial $\mathrm{O}_{2}{ }^{-}$increases with age [56], and endogenous extracellular SOD (superoxide dismutase) may play a role in protection against endothelial dysfunction during aging in mice [57]. In humans, there is an age-related increase in lipoperoxides and a decrease in glutathione peroxidase activity in blood plasma [58]. In 
Figure 2 | Continuity of the mitochondrial germ plasm

(a) Illustration of maintenance of a discrete population of promitochondria (red) as quiescent genetic templates in the female germline, throughout development and between generations. (b) The pre-Weismann view associated with Lamarck, of inheritance of characters acquired by the soma (grey) and transmitted in the germ plasm (white). (c) Weismann's hypothesis of the continuity of the germ plasm from which the soma derives. (d) A view, now discounted, of mitochondria (red) as a component of the soma, and arising from the nucleus (blue). (e) A current view, raising the problem of why there is only maternal transmission of mtDNA, which should transmit age-related mitochondrial mutation if mitochondrial germ plasm (red) ages with the soma (grey). (f) Our view of mitochondrial division of labour between male and female, preserving the Weismann barrier for mitochondrial as well as for nuclear inheritance.
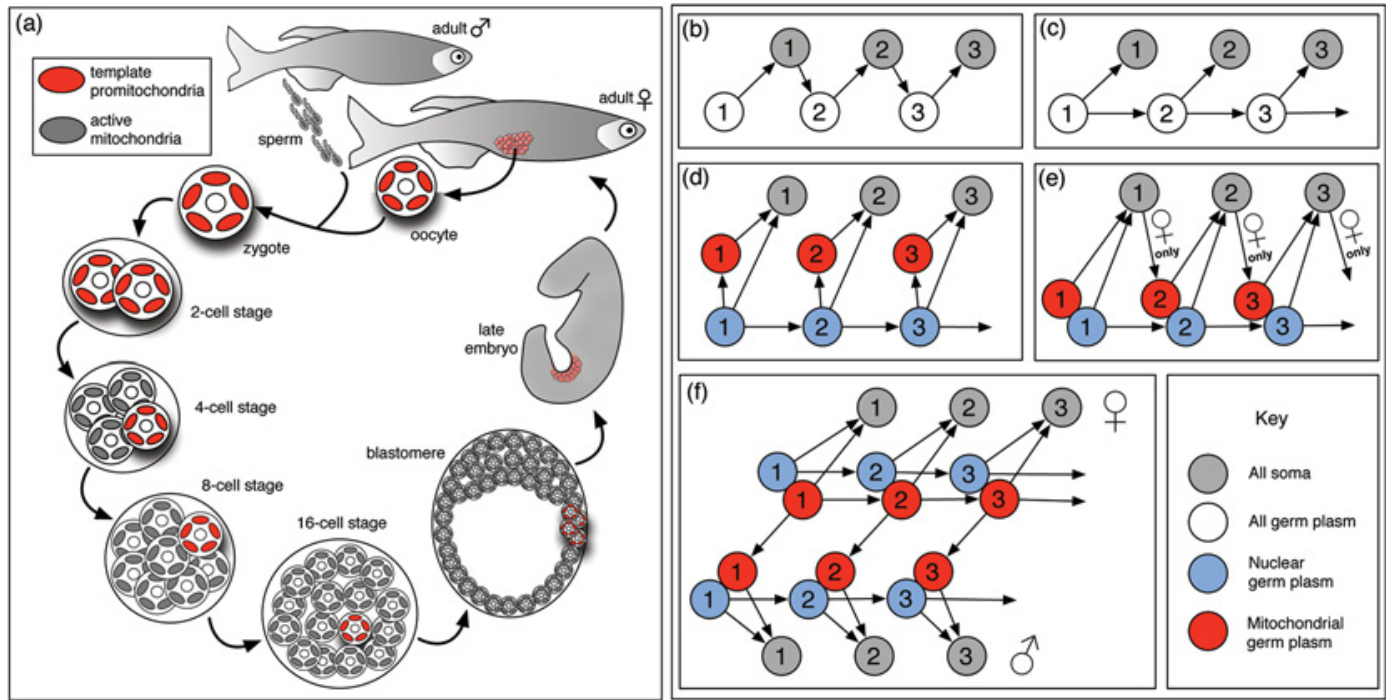

addition, endothelial oxidative stress develops with aging in healthy men and is related to impaired vascular endothelial function $[58,59]$.

\section{Why is acquired mitochondrial mutation not inherited?}

If ROS-induced mtDNA mutation drives the aging process, then it becomes necessary to explain the failure of aging to be inherited, along with the damaged mtDNA. One proposal is that maternally transmitted mitochondria form part of a continuous quiescent lineage of cytoplasmic genetic templates, inactive in transcription and oxidative phosphorylation, and are thus exempt from the aging process [60]. Figure 2(a) illustrates this hypothesis of a selfcontained life cycle of template mitochondria in females. Figure 2(b) diagrammatically distinguishes mitochondrial from nuclear 'germ plasm', a distinction unknown at the time of Weismann's proposed 'central dogma' of the continuity of the germ plasm [61] and of the non-inheritance of somatically acquired characters.

\section{Evidence for quiescent template mitochondria in the female germline}

De Paula et al. [62] report results of experiments designed to test the hypothesis summarized in Figure 2. Using tissue samples from the jellyfish Aurelia aurita (Figure 3a), a member of the ancient animal phylum Cnidaria, de Paula et al. [62] observed decreased transcription of three mitochondrial genes for respiratory chain proteins in ovary, as compared with testis and somatic tissues. The three genes chosen for comparison were nad1, cob and coxI, encoding core protein subunits of the major respiratory complexes I, III and IV, as shown in Figure 1.

Using confocal light microscopy, de Paula et al. [62] also examined and compared mitochondrial membrane potential, as revealed using MitoTracker ${ }^{\circledR}$ Red. It was seen that oocyte mitochondria stand out from those in neighbouring cells, and from those of sperm and somatic cells, in their low-intensity MitoTracker ${ }^{\circledR}$ Red fluorescence. Further results obtained in the course of these experiments [62] are shown in Figures 3(b)-3(d). A corresponding pattern of oocyte mitochondrial quiescence was observed for ROS, as reported fluorescence emission from DCFHDA (2',7'-dichlorodihydrofluorescein diacetate)-oocytes produce fewer ROS when compared with other tissues [62].

Using transmission electron microscopy of tissue sections, de Paula et al. [62] also report that oocyte mitochondria are distinct in size and ultrastructure. Oocytes carry small mitochondria that are simple in internal membrane topology, having few cristae, the invaginations of the mitochondrial inner membrane that carry the respiratory chain complexes and ATP synthase of oxidative phosphorylation. Structurally 
Figure 3 | Evidence for energetically quiescent promitochondria in oocytes

(a) Approximate phylogenetic relation of jellyfish (Cnidaria), Drosophilo (insect; a protostome) and zebrafish (vertebrate; a deuterostome). Confocal microscopy images of ovaries of jellyfish (b), Drosophila (c) and zebrafish (d) showing a marker for intact mitochondria in green (MitoTracker ${ }^{\circledR}$ Green or, in Drosophilo, a mitochondrially imported YFP), and a marker for mitochondrial activity and membrane potential in red (MitoTracker ${ }^{\circledR}$ Red FM). Two distinct mitochondrial populations are highlighted in the zoomed panels: Left: active somatic follicle cell mitochondria, indicated by a blue arrow. Right: quiescent female germline mitochondria, indicated by a white arrow.

(a)

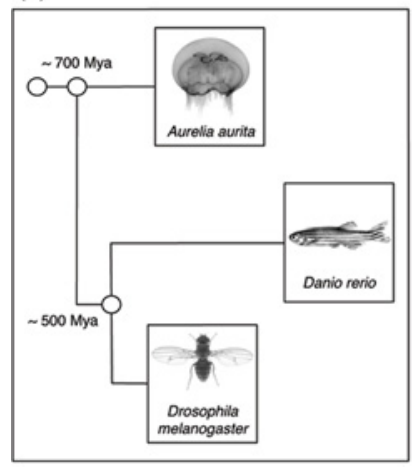

(b)

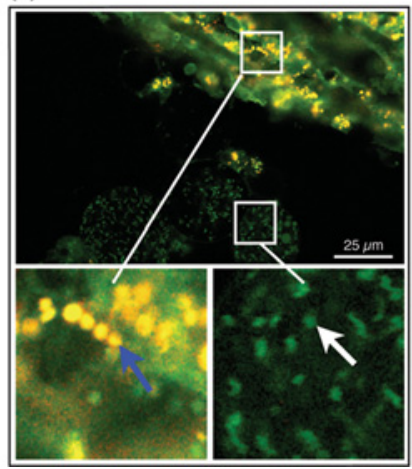

(c)

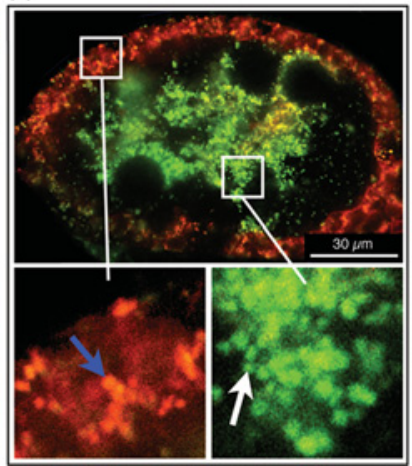

(d)

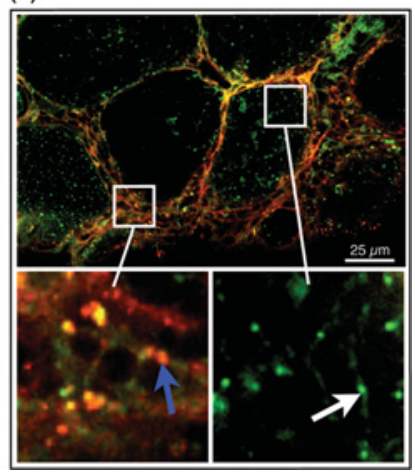

simple mitochondria have been reported to accompany repressed respiratory and complex IV activity in oocytes of the amphibian Xenopus laevis [63]. By analogy with proplastids of plant and algal cells, we use the term promitochondria [60] to describe these organelles.

\section{An evolutionary perspective on mitochondria, separate sexes, and the female germline}

In summary, de Paula et al. [62] conclude that oocyte mitochondria serve as quiescent genetic templates, and are bioenergetically dormant. Fertilization requires sperm motility, and sperm mitochondria are active in ATP synthesis. Elimination of the incoming, paternal, sperm mitochondria from the zygote $[64,65]$ underlies mitochondrial maternal inheritance. This selective elimination, leaving only quiescent promitochondria provided by the oocyte, may explain the non-inheritance of mitochondrial mutation acquired with aging. By permitting a new population of mitochondria to arise from a fresh and uncorrupted mtDNA template in each generation, continuity of a mitochondrial germ plasm may be preserved (Figure 2a).

It remains to be demonstrated that a continuous line of cells, forming the female germline, is maintained from oocyte, through each generation, to oocyte, as illustrated in Figures 2(a) and 2(f). Reversion of functional and differentiated mitochondria to promitochondria at any stage of development would falsify the hypothesis we propose $[60,62]$. Experimental evidence for this reversion, and for discontinuity of promitochondria in the female germline, is a priority for future research. Several lines of evidence suggest a role for mitochondria in sex determination [66]. We propose that complementarity between male and female gametes reconciles the energetics and genetic requirements of mitochondria [60]. We further suggest that this widespread biological division of labour arose with the evolutionary origin of separate sexes $[60,66]$

\section{Acknowledgements}

We thank C.A. Allen, N. Lane, W.F. Martin and J.A. Raven for discussions. We thank F. Missirlis, C.H. Lucas, and R. Ashworth for discussions and for providing the biological samples used in the experiments described in Figure 3.

\section{Funding}

The research is supported by The Leverhulme Trust [grant number F/07 476/AQ] to J.F.A. with co-investigators B. Curran, N. Krauß and A.C. Cuming whom we also thank for discussions.

\section{References}

1 Anderson, S., Bankier, A.T., Barrell, B.G., de Bruijn, M.H., Coulson, A.R. Drouin, J., Eperon, I.C., Nierlich, D.P., Roe, B.A., Sanger, F. et al. (1981) Sequence and organization of the human mitochondrial genome. Nature 290, 457-465

2 Esser, C., Ahmadinejad, N., Wiegand, C., Rotte, C., Sebastiani, F., Gelius-Dietrich, G., Henze, K., Kretschmann, E., Richly, E., Leister, D. et al. (2004) A genome phylogeny for mitochondria among $\alpha$-proteobacteria and a predominantly eubacterial ancestry of yeast nuclear genes. Mol. Biol. Evol. 21, 1643-1660

3 Pesole, G., Allen, J.F., Lane, N., Martin, W., Rand, D.M., Schatz, G. and Saccone, C. (2012) The neglected genome. EMBO Rep. 13, 473-474

4 Calvo, S.E. and Mootha, V.K. (2010) The mitochondrial proteome and human disease. Annu. Rev. Genomics Hum. Genet. 11, 25-44

5 Rhee, H.W., Zou, P., Udeshi, N.D., Martell, J.D., Mootha, V.K., Carr, S.A. and Ting, A.Y. (2013) Proteomic mapping of mitochondria in living cells via spatially restricted enzymatic tagging. Science 339, 1328-1331 
6 Smith, A.C., Blackshaw, J.A. and Robinson, A.J. (2012) MitoMiner: a data warehouse for mitochondrial proteomics data. Nucleic Acids Res. 40 D1160-D1167

7 Dudek, J., Rehling, P. and van der Laan, M. (2013) Mitochondrial protein import: common principles and physiological networks. Biochim. Biophys. Acta 1833, 274-285

8 Schmidt, 0., Pfanner, N. and Meisinger, C. (2010) Mitochondrial protein import: from proteomics to functional mechanisms. Nat. Rev. Mol. Cell Biol. 11, 655-667

9 Ernster, L. and Schatz, G. (1981) Mitochondria: a historical review. J. Cell Biol. 91, S227-S255

10 Rich, P.R. (2003) The molecular machinery of Keilin's respiratory chain. Biochem. Soc. Trans. 31, 1095-1105

11 Allen, J.F. (1993) Control of gene-expression by redox potential and the requirement for chloroplast and mitochondrial genomes. J. Theor. Biol. 165, 609-631

12 Allen, J.F. (2003) Why chloroplasts and mitochondria contain genomes. Comp. Funct. Genomics 4, 31-36

13 Allen, J.F. (2003) The function of genomes in bioenergetic organelles Philos. Trans. R. Soc. London Ser. B 358, 19-37

14 Pfannschmidt, T., Nilsson, A. and Allen, J.F. (1999) Photosynthetic contro of chloroplast gene expression. Nature 397, 625-628

15 Allen, J.F. and Pfannschmidt, T. (2000) Balancing the two photosystems: photosynthetic electron transfer governs transcription of reaction centre genes in chloroplasts. Philos. Trans. R. Soc. London Ser. B 355 1351-1357

16 Puthiyaveetil, S., Kavanagh, T.A., Cain, P., Sullivan, J.A., Newell, C.A., Gray, J.C., Robinson, C., van der Giezen, M., Rogers, M.B. and Allen, J.F. (2008) The ancestral symbiont sensor kinase CSK links photosynthesis with gene expression in chloroplasts. Proc. Natl. Acad. Sci. U.S.A. 105 10061-10066

17 Puthiyaveetil, S., Ibrahim, I.M., Jelicic, B., Tomasic, A., Fulgosi, H. and Allen, J.F. (2010) Transcriptional control of photosynthesis genes: the evolutionarily conserved regulatory mechanism in plastid genome function. Genome Biol. Evol. 2, 888-896

18 Puthiyaveetil, S., Ibrahim, I.M. and Allen, J.F. (2012) Oxidation-reduction signalling components in regulatory pathways of state transitions and photosystem stoichiometry adjustment in chloroplasts. Plant Cell Environ. 35, 347-359

19 Allen, J.F., Santabarbara, S., Allen, C.A. and Puthiyaveetil, S. (2011) Discrete redox signaling pathways regulate photosynthetic light-harvesting and chloroplast gene transcription. PLOS ONE 6, e26372

20 Shimizu, M., Kato, H., Ogawa, T., Kurachi, A., Nakagawa, Y. and Kobayashi, H. (2010) Sigma factor phosphorylation in the photosynthetic control of photosystem stoichiometry. Proc. Natl. Acad. Sci. U.S.A. 107, 10760-10764

21 Puthiyaveetil, S., Ibrahim, I.M. and Allen, J.F. (2013) Evolutionary rewiring: a modified prokaryotic gene-regulatory pathway in chloroplasts. Philos. Trans. R. Soc. London Ser. B 368, 20120260

22 Allen, C.A. and Allen, J.F. (2008) Redox effects on chloroplast protein synthesis and phosphorylation. In Photosynthesis. Energy from the Sun 14th International Congress on Photosynthesis (Allen, J.F., Gantt, E., Golbeck, J.H. and Osmond, B., eds), pp. 903-907, Springer, Heidelberg

23 Allen, C.A., Håkansson, G. and Allen, J.F. (1995) Redox conditions specify the proteins synthesized by isolated chloroplasts and mitochondria. Redox Rep. 1, 119-123

24 Escobar Galvis, M.L., Allen, J.F. and Håkansson, G. (1998) Protein synthesis by isolated pea mitochondria is dependent on the activity of respiratory complex II. Curr. Genet. 33, 320-329

25 Wilson, S.B., Davidson, G.S., Thomson, L.M. and Pearson, C.K. (1996) Redox control of RNA synthesis in potato mitochondria. Eur. J. Biochem. 242, 81-85

26 de Paula, W.B.M., Allen, J.F. and van der Giezen, M. (2012) Mitochondria, hydrogenosomes and mitosomes in relation to the CoRR hypothesis for genome function and evolution. In Organelle Genetics (Bullerwell, C.E., ed.), pp. 105-119, Springer-Verlag, Berlin

27 van der Giezen, M. (2009) Hydrogenosomes and mitosomes: conservation and evolution of functions. J. Eukaryotic Microbiol. 56, 221-231

28 Clark, C. and Roger, A. (1995) Direct evidence for secondary loss of mitochondria in Entomoebo histolytico. Proc. Natl. Acad. Sci. U.S.A 92 6518-6521

29 Tovar, J., León-Avila, G., Sánchez, L., Sutak, R., Tachezy, J., van der Giezen, M., Hernández, M., Müller, M. and Lucocq, J. (2003) Mitochondrial remnant organelles of Giardia function in iron-sulphur protein maturation. Nature 426, 172-176
30 Lill, R. and Mühlenhoff, U. (2005) Iron-sulfur protein biogenesis in eukaryotes. Trends Biochem. Sci. 30, 133-141

31 Lindmark, D.G. and Muller, M. (1973) Hydrogenosome, a cytoplasmic organelle of the anaerobic flagellate Trichomonos foetus, and its role in pyruvate metabolism. J. Biol. Chem 248, 7724-7728

32 Muller, M. (1993) The hydrogenosome. J. Gen. Microbiol. 139 2879-2889

33 Bui, E.T., Bradley, P.J. and Johnson, P.J. (1996) A common evolutionary origin for mitochondria and hydrogenosomes. Proc. Natl. Acad. Sci. U.S.A. 93, 9651-9656

34 Muller, M., Mentel, M., van Hellemond, I.J., Henze, K., Woehle, C., Gould, S.B., YU, R.Y., van der Giezen, M., Tielens, A.G.M. and Martin, W.F. (2012) Biochemistry and evolution of anaerobic energy metabolism in eukaryotes. Microbiol. Mol. Biol. Rev. 76, 444-495

35 Allen, C.A., van der Giezen, M. and Allen, J.F. (2007) Origin, function and transmission of mitochondria. In Origins of Mitochondria and Hydrogenosomes (Martin, W.F. and Müller, M., eds), pp. 39-56, Springer-Verlag, Berlin

36 Lane, N. and Martin, W. (2010) The energetics of genome complexity. Nature 467, 929-934

37 Puthiyaveetil, S. and Allen, J.F. (2009) Chloroplast two-component systems: evolution of the link between photosynthesis and gene expression. Proc. R. Soc. London Ser. B 276, 2133-2145

38 Mavrianos, J., Berkow, E.L., Desai, C., Pandey, A., Batish, M., Rabadi, M.)., Barker, K.S., Pain, D., Rogers, P.D., Eugenin, E.A. and Chauhan, N. (2013) Mitochondrial two-component signaling systems in Condida albicans. Eukaryotic cell 12, 913-922

39 Wuichet, K., Cantwell, B.J. and Zhulin, I.B. (2010) Evolution and phyletic distribution of two-component signal transduction systems. Curr. Opin. Microbiol. 13, 219-225

40 Allen, J.F. (1993) Redox control of transcription: sensors, response regulators, activators and repressors. FEBS Lett. 332, 203-207

41 Martin, W. and Muller, M. (1998) The hydrogen hypothesis for the first eukaryote. Nature 392, 37-41

42 Armstrong, J.S., Whiteman, M., Yang, H. and Jones, D.P. (2004) The redox regulation of intermediary metabolism by a superoxide-aconitase rheostat. BioEssays 26, 894-900

43 Douarre, C., Sourbier, C., Dalla Rosa, I., Das, B.B., Redon, C.E., Zhang, H.L., Neckers, L. and Pommier, Y. (2012) Mitochondrial topoisomerase I is critical for mitochondrial integrity and cellular energy metabolism. PLos ONE 7, e41094

44 Tan, X.Y., Liu, X.L., Wang, W., Jia, D.J., Chen, L.Q., Zhang, X.Q. and Ye, D. (2010) Mutations in the Arabidopsis nuclear-encoded mitochondrial phage-type RNA polymerase gene RPOTm led to defects in pollen tube growth, female gametogenesis and embryogenesis. Plant Cell Physiol. 51, 635-649

45 Chance, B., Sies, H. and Boveris, A. (1979) Hydroperoxide metabolism in mammalian organs. Physiol. Rev. 59, 527-605

46 Chen, Q., Vazquez, E.J., Moghaddas, S., Hoppel, C.L. and Lesnefsky, E.J. (2003) Production of reactive oxygen species by mitochondria: central role of complex III. J. Biol. Chem. 278, 36027-36031

47 Allen, J.F. and Raven, J.A. (1996) Free-radical-induced mutation vs redox regulation: costs and benefits of genes in organelles. J. Mol. Evol. 42 482-492

48 Ernster, L. (1994) The merger of bioenergetics and molecular-biology. Biochem. Soc. Trans. 22, 253-265

49 Harman, D. (1972) The biologic clock: the mitochondria? J. Am. Geriatr. Soc. 20, 145-147

50 Ames, B.N., Shigenaga, M.K. and Hagen, T.M. (1995) Mitochondrial decay in aging. Biochim. Biophys. Acta 1271, 165-170

51 Wallace, D.C. (2010) Mitochondrial DNA mutations in disease and aging. Environ. Mol. Mutagen. 51, 440-450

52 Afanas'ev, I. (2010) Signaling and damaging functions of free radicals in aging-free radical theory, hormesis, and TOR. Aging Dis. 1, 75-88

53 Jacobs, H.T. (2003) The mitochondrial theory of aging: dead or alive? Aging Cell 2,11-17

54 Sasaki, T., Unno, K., Tahara, S., Shimada, A., Chiba, Y., Hoshino, M. and Kaneko, T. (2008) Age-related increase of superoxide generation in the brains of mammals and birds. Aging Cell 7, 459-469

55 Miyazawa, M., Ishii, T., Yasuda, K., Noda, S., Onouchi, H., Hartman, P.S. and Ishii, N. (2009) The role of mitochondrial superoxide anion $\left(\mathrm{O}_{2}{ }^{-}\right)$on physiological aging in (57BL/6) mice. J. Radiat. Res. 50, 73-83

56 Jacobson, A., Yan, C., Gao, Q., Rincon-Skinner, T., Rivera, A., Edwards, J., Huang, A., Kaley, G. and Sun, D. (2007) Aging enhances pressure-induced arterial superoxide formation. Am. J. Physiol. Heart Circ. Physiol. 293, H1344-H1350 
57 Lund, D.D., Chu, Y., Miller, J.D. and Heistad, D.D. (2009) Protective effect of extracellular superoxide dismutase on endothelial function during aging. Am. J. Physiol. Heart Circ. Physiol. 296, H1920-H1925

58 Mendoza-Nunez, V.M., Ruiz-Ramos, M., Sanchez-Rodriguez, M.A., Retana-Ugalde, R. and Munoz-Sanchez, J.L. (2007) Aging-related oxidative stress in healthy humans. Tohoku J. Exp. Med. 213, 261-268

59 Donato, A.J., Eskurza, I., Silver, A.E., Levy, A.S., Pierce, G.L., Gates, P.E. and Seals, D.R. (2007) Direct evidence of endothelial oxidative stress with aging in humans: relation to impaired endothelium-dependent dilation and upregulation of nuclear factor- $\kappa$ B. Circ. Res. 100, 1659-1666

60 Allen, J.F. (1996) Separate sexes and the mitochondrial theory of ageing. J. Theor. Biol. 180, 135-140

61 Weismann, A. (1889) Essays Upon Heredity, Clarendon Press, Oxford

62 de Paula, W.B.M., Lucas, C.H., Agip, A.N.-A., Vizcay-Barrena, G. and Allen, J.F. (2013) Energy, ageing, fidelity and sex: oocyte mitochondrial DNA as a protected genetic template. Philos. Trans. R. Soc. London Ser. B $\mathbf{3 6 8}$ 20120263
63 Kogo, N., Tazaki, A., Kashino, Y., Morichika, K., Orii, H., Mochii, M. and Watanabe, K. (2011) Germ-line mitochondria exhibit suppressed respiratory activity to support their accurate transmission to the next generation. Dev. Biol. 349, 462-469

64 Al Rawi, S., Louvet-Vallee, S., Djeddi, A., Sachse, M., Culetto, E., Hajjar, C., Boyd, L., Legouis, R. and Galy, V. (2011) Postfertilization autophagy of sperm organelles prevents paternal mitochondrial DNA transmission. science 334, 1144-1147

65 Deluca, S.Z. and O'Farrell, P.H. (2013) Barriers to male transmission of mitochondrial DNA in sperm development. Dev. Cell 22, 660-668

66 Mittwoch, U. (2013) Sex determination. EMBO Rep. 14, 588-592

Received 4 June 2013

doi:10.1042/BST20130106 